\title{
ASYMPTOTIC DICHOTOMY FOR NONOSCILLATORY SOLUTIONS OF A NONLINEAR DIFFERENCE EQUATION
}

Abstract. A nonlinear difference equation involving the maximum function is studied. We derive sufficient conditions in order that eventually positive or eventually negative solutions tend to zero or to positive or negative infinity.

Nonlinear difference equations involving three or more functional values of the state variable are important as they appear naturally as discrete analogs and as numerical schemes of differential equations which model various natural phenomena. For an introductory exposition, the readers may consult, e.g., Kocic and Ladas [2]. In this paper, we are concerned with the nonlinear difference equation

$$
\Delta^{2}\left(x_{n}-p_{n} x_{n-\tau}\right)+q_{n} \max _{n-\sigma \leq s \leq n} x_{s}=0, \quad n=0,1, \ldots,
$$

where $\tau>0, \sigma \geq 0$, and $\left\{p_{n}\right\}_{n=0}^{\infty},\left\{q_{n}\right\}_{n=0}^{\infty}$ are real sequences. For $\sigma=0$, the above equation reduces to the linear equation

$$
\Delta^{2}\left(x_{n}-p_{n} x_{n-\tau}\right)+q_{n} x_{n}=0, \quad n=0,1,2, \ldots,
$$

which has been studied by Zhang and Cheng [5]. For $\sigma=0$ and $\left\{p_{n}\right\} \equiv 0$, (1) reduces further to the second order linear difference equation

$$
\Delta^{2} x_{n}+q_{n} x_{n}=0, \quad n=0,1,2, \ldots,
$$

which has been studied by a number of authors.

Let $\mu=\max \{\tau, \sigma\}$. If a real sequence $x=\left\{x_{n}\right\}_{n=-\mu}^{\infty}$ satisfies the functional relation defined by (1), then it is said to be a solution of (1). Since

1991 Mathematics Subject Classification: Primary 39A10.

Key words and phrases: difference equation, eventually positive solution, asymptotic dichotomy.

Research of S. S. Cheng partially supported by the National Science Council of R.O.C. 
(1) is a recurrence relation, it is not difficult to see that when initial conditions $x_{-\mu}, x_{-\mu+1}, \ldots, x_{1}$ are given, we can successively calculate $x_{2}, x_{3}, \ldots$ in a unique manner. An existence and uniqueness theorem can thus be formulated and proved.

We will be interested in the asymptotic behavior of eventually positive or eventually negative solutions of equation (1). In particular, sufficient conditions for such solutions to tend either to zero or to infinity are derived. Before doing so, we first discuss the question of existence of eventually positive or eventually negative solutions.

First of all, eventually positive (and hence eventually negative) solutions of (3) exist when $\left\{q_{n}\right\}$ satisfies appropriate conditions (see e.g. $[1,3,4]$ ). Furthermore, an eventually positive nondecreasing solution of (2) is also an eventually positive solution of (1). Existence criteria for eventually positive nondecreasing solutions of (2) can be found in [1,3-5]. As another example, the constant sequence $\{1\}$ is a solution of the equation

$$
\Delta^{2}\left(x_{n}-n(n-1) x_{n-\tau}\right)+2 \max _{n-\sigma \leq s \leq n} x_{s}=0, \quad n=0,1,2, \ldots
$$

For $p_{n} \equiv p$ and $q_{n} \equiv q$, we can also look for solutions of the form $\pm \lambda^{-n}$. Care must be taken, however, to distinguish the cases $\lambda \in(0,1)$ and $\lambda>1$. Suppose we try to look for an eventually positive solution of the form $\lambda^{-n}$ where $0<\lambda<1$. Substitution of $\lambda^{-n}$ into (1) leads to the equation

$$
\Gamma(\lambda) \equiv\left(1-p \lambda^{\tau}\right)(1-1 / \lambda)^{2}+q=0 .
$$

Since $\Gamma\left(0^{+}\right)=\infty$ and $\Gamma\left(1^{-}\right)=q$, if we assume that $q<0$, then $\Gamma(\lambda)$ will have a root $\lambda_{*}$ in $(0,1)$ and hence $\lambda_{*}^{-n}$ is a desired solution. Similarly, if we look for a solution of the form $\lambda^{-n}$ where $\lambda>1$, then substitution of $\lambda^{-n}$ into (1) leads to

$$
\Psi(\lambda)=\left(1-p \lambda^{\tau}\right)(1-1 / \lambda)^{2}+q \lambda^{\sigma}=0 .
$$

Since $\Psi\left(1^{+}\right)=q$, any set of conditions on $p, q, \tau$ and $\sigma$ which yields $q \lim _{\lambda \rightarrow \infty} \Psi(\lambda)<0$ will also yield a desired solution. For instance, the conditions $\tau=\sigma$ and $q p>0$ will do the job. We can also look for eventually negative solutions of (1) in a similar manner. For instance, if $p_{n} \equiv p>0$, $q_{n} \equiv q>0$ and $\tau>0$, then (1) has an eventually negative solution of the form $-\lambda^{-n}$ where $\lambda>1$.

Now that we have demonstrated the existence of an eventually positive or an eventually negative solution $\left\{x_{n}\right\}$, we will show that its "companion" sequence $\left\{z_{n}\right\}$ defined by

$$
z_{n}=x_{n}-p_{n} x_{n-\tau}, \quad n=0,1,2, \ldots,
$$

has the following monotonicity properties. 
LEMma 1. Suppose that there is a positive number $p$ such that $0 \leq p_{n} \leq p$ for $n \geq 0$, and that $q_{n} \geq 0$ for $n \geq 0$ as well as

$$
\sum_{n=0}^{\infty} q_{n}=\infty
$$

Then for any eventually positive solution $\left\{x_{n}\right\}$ of (1), its companion sequence $\left\{z_{n}\right\}$ defined by (5) satisfies either

(i) $z_{n}<0, \Delta z_{n}<0, \Delta^{2} z_{n} \leq 0$ for all large $n$, and $\lim _{n \rightarrow \infty} z_{n}=$ $\lim _{n \rightarrow \infty} \Delta z_{n}=-\infty$; or

(ii) $z_{n}<0, \Delta z_{n}>0, \Delta^{2} z_{n} \leq 0$ for all large $n$, and $\lim _{n \rightarrow \infty} z_{n}=$ $\lim _{n \rightarrow \infty} \Delta z_{n}=0$.

Proof. In view of (1) and (6), $\left\{\Delta^{2} z_{n}\right\}$ is eventually nonpositive and not identically zero for all large $n$. Hence $\lim _{n \rightarrow \infty} \Delta z_{n}=-\infty$ or $\lim _{n \rightarrow \infty} \Delta z_{n}$ $=L$. In the former case, clearly conclusion (i) must hold. In the latter, we consider three subcases: (a) $L<0$, (b) $L=0$, and (c) $L>0$. If $L<0$, then $\lim _{n \rightarrow \infty} z_{n}=-\infty$. But then in view of

$$
z_{n}=x_{n}-p_{n} x_{n-\tau}>-p_{n} x_{n-\tau} \geq-p x_{n-\tau},
$$

we see that $\lim _{n \rightarrow \infty} x_{n}=\infty$, so that by summing (1) from a sufficiently large integer $N$ to $n-1$, we have

$$
\Delta z_{n}=\Delta z_{N}-\sum_{j=N}^{n-1} q_{j} \max _{j-\sigma \leq s \leq j} x_{s} \rightarrow-\infty
$$

as $n \rightarrow \infty$. This is a contradiction. By a similar reasoning, the case $L>0$ is also impossible. Finally, suppose $\lim _{n \rightarrow \infty} \Delta z_{n}=0$. Clearly $\Delta z_{n}>0$ for all large $n$, and hence $\lim _{n \rightarrow \infty} z_{n}$ is either $\infty,>0$, or $\leq 0$. If it is either infinite or positive, then $x_{n} \geq x_{n}-p_{n} x_{n-\tau} \geq z_{n} \geq \Gamma>0$ for all large $n$, so that $\Delta z_{n} \rightarrow-\infty$ as before. If $\lim _{n \rightarrow \infty} z_{n}=M<0$, then $M \geq z_{n}>-p_{n} x_{n-\tau} \geq-p x_{n-\tau}$ for all large $n$. Hence $x_{n-\tau}>-M / p>0$ for all large $n$, so that $\Delta z_{n} \rightarrow-\infty$ as before. The only case possible is $\lim _{n \rightarrow \infty} z_{n}=0$. The proof is complete.

We remark that under the same conditions as in Lemma 1, we can obtain a dual statement: for an eventually negative solution $\left\{x_{n}\right\}$ of (1), its companion sequence $\left\{z_{n}\right\}$ satisfies either (i) $z_{n}>0, \Delta z_{n}>0, \Delta^{2} z_{n} \geq 0$ for all large $n$, and $\lim _{n \rightarrow \infty} z_{n}=\lim _{n \rightarrow \infty} \Delta z_{n}=\infty$; or (ii) $z_{n}>0, \Delta z_{n}<0$, $\Delta^{2} z_{n} \geq 0$ for all large $n$, and $\lim _{n \rightarrow \infty} z_{n}=\lim _{n \rightarrow \infty} \Delta z_{n}=0$.

We remark further that if in addition to the assumptions of Lemma 1 we also assume that there is a positive integer $N$ such that

$$
p_{N+j \tau} \leq 1, \quad j=0,1,2, \ldots,
$$


then the conclusion (ii) must hold for an eventually positive solution of (1). Otherwise there is a positive number $\alpha$ and an integer $J$ such that $z_{n}<-\alpha$ for $n \geq J$. Thus

$$
x_{n}=z_{n}+p_{n} x_{n-\tau}<-\alpha+p_{n} x_{n-\tau}, \quad n \geq J .
$$

Pick an integer $M$ so large that $N+M \tau \geq J$. Then for any integer $k \geq 1$,

$$
\begin{aligned}
x_{N+M \tau+k \tau} & =z_{N+M \tau+k \tau}+p_{N+M \tau+k \tau} x_{N+M \tau+(k-1) \tau} \\
& <-\alpha+x_{N+M \tau+(k-1) \tau}<\ldots<-k \alpha+x_{N+M \tau},
\end{aligned}
$$

contrary to the fact that $\left\{x_{n}\right\}$ is eventually positive.

LEMMA 2. In addition to the assumptions of Lemma 1, assume that there is an integer $N$ such that (7) holds. Then the companion sequence $\left\{z_{n}\right\}$ of an eventually positive solution $\left\{x_{n}\right\}$ of (1) satisfies conclusion (ii) of Lemma 1.

As a dual result, we easily see that under the conditions of Lemma 2, the companion sequence $\left\{z_{n}\right\}$ of an eventually negative solution will satisfy $z_{n}>$ $0, \Delta z_{n}<0, \Delta^{2} z_{n} \geq 0$ for all large $n$, as well as $\lim _{n \rightarrow \infty} z_{n}=\lim _{n \rightarrow \infty} \Delta z_{n}$ $=0$.

We now show that under the conditions of Lemma 1, an eventually positive solution either diverges to $\infty$ or else its lower limit is zero.

THEOREM 1. Let the conditions of Lemma 1 hold. If $\left\{x_{n}\right\}$ is an eventually positive solution of (1), then either $\lim _{n \rightarrow \infty} x_{n}=\infty$ or $\liminf _{n \rightarrow \infty} x_{n}$ $=0$.

P r o of. If conclusion (i) of Lemma 1 holds, then

$$
z_{n}>-p_{n} x_{n-\tau} \geq-p x_{n-\tau}
$$

for all large $n$. But since $\lim _{n \rightarrow \infty} z_{n}=-\infty$, we must have $\lim _{n \rightarrow \infty} x_{n}=\infty$. If conclusion (ii) holds, then by summing (1) from a sufficiently large integer $N$, we see that

$$
\Delta z_{n+1}+\sum_{i=N}^{n} q_{i} \max _{i-\sigma \leq s \leq i} x_{s}=\Delta z_{N}, \quad n \geq N .
$$

Since $\lim _{n \rightarrow \infty} \Delta z_{n}=0$, we see that

$$
\sum_{i=N}^{\infty} q_{i} \max _{i-\sigma \leq s \leq i} x_{s}<\infty
$$

which implies $\liminf \operatorname{in}_{n \rightarrow \infty} x_{n}=0$. The proof is complete.

We remark that if $\left\{p_{n}\right\}$ is unbounded, then the conclusion of Theorem 1 may not hold. As an example, the solution $\{1\}$ of (4) does not satisfy the alternative of Theorem 1 . 
We remark further that since the conditions of Lemma 2 prevent conclusion (i) of Lemma 1 from happening, they also rule out the conclusion $\lim _{n \rightarrow \infty} x_{n}=\infty$ of Theorem 1 .

TheOrem 2. Suppose the conditions of Lemma 2 hold. If $\left\{x_{n}\right\}$ is an eventually positive solution of (1), then $\liminf _{n \rightarrow \infty} x_{n}=0$.

It is desirable to strengthen the alternative in Theorem 1 . One way to achieve this is to replace the condition (6) by a stronger one.

THEOREM 3. Suppose that there is a positive number $p$ such that $0 \leq$ $p_{n} \leq p$ for $n \geq 0$, and that $q_{n} \geq q>0$ for $n \geq 0$. Then for any eventually positive solution $\left\{x_{n}\right\}$ of (1), either $\lim _{n \rightarrow \infty} x_{n}=\infty$ or $\lim _{n \rightarrow \infty} x_{n}=0$.

Proof. If conclusion (i) of Lemma 1 holds, then $z_{n}>-p_{n} x_{n-\tau} \geq$ $-p x_{n-\tau}$ for all large $n$. But since $\lim _{n \rightarrow \infty} z_{n}=-\infty$, we must have $\lim _{n \rightarrow \infty} x_{n}=\infty$. Suppose (ii) of Lemma 1 holds. If $\left\{x_{n}\right\}$ does not converge to zero, then there is a sequence $\{n(i)\}_{i=0}^{\infty}$ of integers such that $n(i+1)-n(i)>\sigma$ and $x_{n(i)}>\delta>0$ for $i=0,1,2, \ldots$ As seen in the proof of Theorem 1, we have

On the other hand,

$$
\sum_{n=n(0)}^{\infty} q_{n} \max _{n-\sigma \leq s \leq n} x_{s}<\infty
$$

$$
\sum_{n=n(0)}^{\infty} q_{n} \max _{n-\sigma \leq s \leq n} x_{s} \geq \sum_{i=1}^{\infty} \sum_{j=n(i)}^{n(i)+\sigma} q_{j} \max _{j-\sigma \leq s \leq j} x_{s} \geq \sum_{i=1}^{\infty} q \delta(\sigma+1)=\infty,
$$

which is a contradiction.

We remark that under the same conditions as in Theorem 3, an eventually negative solution may have an infinite lower limit and also a zero upper limit simultaneously. As an example, the equation

$$
\Delta^{2}\left(x_{n}-4 x_{n-2}\right)+\frac{15}{4} \max _{n-2 \leq s \leq n} x_{s}=0, \quad n=0,1,2, \ldots,
$$

satisfies the assumptions of Theorem 3 , yet it has an eventually negative solution $\left\{x_{n}\right\}=\left\{-2^{n}\left(1+(-1)^{n}\right)-2^{-n}\right\}$ which satisfies $\lim \sup _{n \rightarrow \infty} x_{n}=0$ and $\liminf _{n \rightarrow \infty} x_{n}=-\infty$.

However, if we impose the additional assumption that there is an integer $N$ such that (7) holds, then as seen in Lemma 2, $\left\{x_{n}\right\}$ cannot diverge to $\infty$.

THEOREM 4. In addition to the assumptions of Theorem 3, assume that there is an integer $N$ such that (7) holds. Then every eventually positive solution of (1) converges to 0 .

We remark that under the conditions Theorem 4, eventually negative solutions of (1) may not converge to 0 . An example is provided by the 
equation

$$
\Delta^{2}\left(x_{n}-x_{n-2}\right)+\frac{3}{4} \max _{n-2 \leq s \leq n} x_{s}=0, \quad n=0,1,2, \ldots,
$$

which has a divergent and eventually negative solution $\left\{-1-(-1)^{n}-2^{-n}\right\}$.

So far we have been concerned with conditions which are sufficient for eventually positive solutions to have zero lower limits. Our last result provides a sufficient condition for such solutions to diverge.

THEOREM 5. In addition to the assumptions of Lemma 1, assume that $\sigma>\tau$ and

$$
\limsup _{n \rightarrow \infty} \sum_{i=n+\tau-\sigma}^{n-1}(i+1+\sigma-n-\tau) q_{i}>p .
$$

Then every eventually positive solution of (1) diverges to $\infty$ and every eventually negative solution diverges to $-\infty$.

P r o of. Let $\left\{x_{n}\right\}$ be an eventually positive solution of (1). If conclusion (ii) of Lemma 1 holds, then $z_{n}<0, \Delta z_{n}>0$ and

$$
z_{n}=x_{n}-p_{n} x_{n-\tau}>-p_{n} x_{n-\tau} \geq-p x_{n-\tau}
$$

for all large $n$. In view of (1), we see further that

$$
\begin{aligned}
\Delta^{2} z_{n}-\frac{q_{n}}{p} z_{n-(\sigma-\tau)} & \leq \Delta^{2} z_{n}+q_{n} \max _{n-\sigma \leq s \leq n} \frac{-z_{s+\tau}}{p} \\
& \leq \Delta^{2} z_{n}+q_{n} \max _{n-\sigma \leq s \leq n} x_{s}=0
\end{aligned}
$$

for all large $n$. Writing $z_{n}$ as $-u_{n}$, we see that $\left\{u_{n}\right\}$ is positive decreasing and

$$
\Delta^{2} u_{n} \geq \frac{q_{n}}{p} u_{n-(\sigma-\tau)}
$$

for all large $n$. Hence

$$
\sum_{t=n-(\sigma-\tau)}^{n-1} \sum_{i=t}^{n-1} \Delta^{2} u_{i} \geq \sum_{t=n-(\sigma-\tau)}^{n-1} \sum_{i=t}^{n-1} \frac{q_{i}}{p} u_{i-(\sigma-\tau)}
$$

or

$$
(\sigma-\tau) \Delta u_{n}-u_{n}+u_{n-(\sigma-\tau)} \geq \sum_{i=n-(\sigma-\tau)}^{n-1}(i+1-n+\sigma-\tau) \frac{q_{i}}{p} u_{i-(\sigma-\tau)}
$$

for all large $n$. Since $\left\{u_{n}\right\}$ is eventually positive decreasing, we see that

$$
u_{n-(\sigma-\tau)} \geq \sum_{i=n-(\sigma-\tau)}^{n-1}(i+1-n+\sigma-\tau) \frac{q_{i}}{p} u_{i-(\sigma-\tau)},
$$


and hence,

$1>\sum_{i=n-(\sigma-\tau)}^{n-1}(i+1-n+\sigma-\tau) \frac{q_{i}}{p} \cdot \frac{u_{i-(\sigma-\tau)}}{u_{n-(\sigma-\tau)}}>\sum_{i=n-(\sigma-\tau)}^{n-1}(i+1-n+\sigma-\tau) \frac{q_{i}}{p}$,

contrary to (8). The case where $\left\{x_{n}\right\}$ is eventually negative is similar. The proof is complete.

\section{References}

[1] S. S. Cheng and B. G. Zhang, Monotone solutions of a class of nonlinear difference equations, Comput. Math. Appl. 28 (1994), 71-79.

[2] V. L. Kocic and G. Ladas, Global Behavior of Nonlinear Difference Equations of Higher Order with Applications, Math. Appl. 256, Kluwer, 1993.

[3] H. J. Li and S. S. Cheng, Asymptotically monotone solutions of a nonlinear difference equation, Tamkang J. Math. 24 (1993), 269-282.

[4] B. Liu and S. S. Cheng, Positive solutions of second order nonlinear difference equations, J. Math. Anal. Appl. 204 (1996), 482-493.

[5] B. G. Zhang and S. S. Cheng, Oscillation criteria and comparison theorems for delay difference equations, Fasc. Math. 25 (1995), 13-32.

Guang Zhang

Department of Mathematics

Datong Advanced College

Datong, Shanxi 037008

P.R. China
Sui Sun Cheng

Department of Mathematics Tsing Hua University Hsinchu, Taiwan 30043 R.O.C. 